\title{
Process Development for Active Pharmaceutical Ingredients Following a Developmental Cascade
}

\author{
Carmen Adler, Jürg Brunner, Claudia Fichtner, Peter Küng, Michael K. Levis, Hans-Rudolf Ruchti, \\ Anders Sjöberg, and Beat Weber*
}

\begin{abstract}
The developmental process for drug substances, as carried out in a medium-sized company is described. A cascade approach, ranging from route selection to commercial production and the corresponding intermediate steps, is discussed. A concerted teamwork is essential for optimal results, and the roles and activities of the team members are outlined.
\end{abstract}

Keywords: API · Developmental cascade · Drug substance · Process development

\section{Introduction}

The developmental process for APIs (Active Pharmaceutical Ingredients, also called drug substances) is a most challenging task. Contradicting goals must be balanced, and these include the consideration of quality, safety, robustness, costs and time constraints (Fig.1). The workflow outlined here defines the methodology used at Siegfried Ltd, Zofingen. The company manufactures APIs (and key intermediates) on an exclusive basis for a number of pharmaceutical companies, as well as for the generic pharmaceutical industry.

All aspects of the API that influence its identity, strength, purity, and quality are awarded top attention. Because the drugs are prescribed to patients, the health authorities have established guidelines [1] for manufacturers to follow that address the quality of the production process. The

\footnotetext{
${ }^{*}$ Correspondence: Dr. B. Weber

Siegfried Ltd

Process Research

Untere Brühlstrasse 4

$\mathrm{CH}-4800$ Zofingen

Tel.: +41627461258

Fax: +41627461205

E-Mail: beat.weber@siegfried.ch

www.siegfried.ch
}

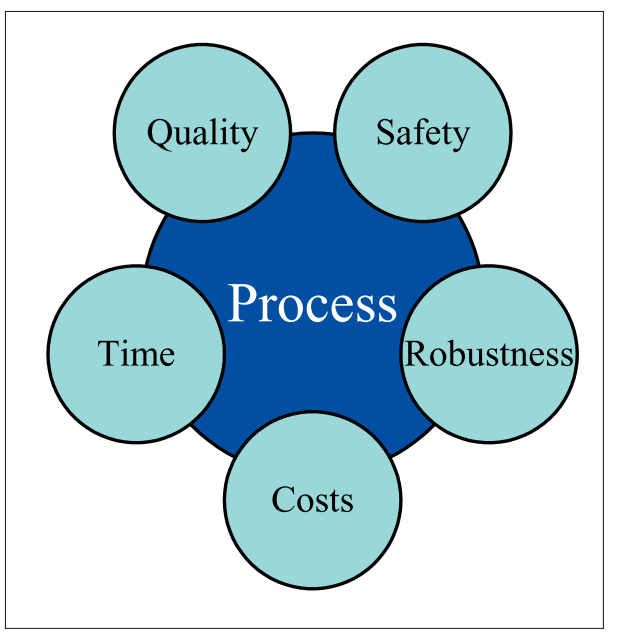

Fig. 1. Perimeter of process optimization

safety of the process is a target dictated by a sense of responsibility: At no time may a process pose a significant risk to operators, equipment or the environment.

To ensure smooth production, a process must be as sturdy and robust as possible. The ranges set for specific parameters (e.g. temperature, reaction time, concentration), and within which the process can be operated without failure, determine the robustness of the process. Naturally, costs must be kept under control, both in the developmental stage of a drug and during the commercial manufacturing process once the drug is on the market. In the early stages of drug development, resource allocation for individual projects is limited; a majority of these candidates will never reach the market having failed in clinical trials. Later, however, during the commercialization phase, the drug must be competitively priced and its manufacturing process optimized. Time spent during the clinical development determines the entry date of a drug to market and, eventually, the lifespan of its patent protection. It is thus important that the development time of the API synthesis does not restrict the drug development process.

Fig. 2 outlines the developmental cascade. At Siegfried, this has proven to be a versatile scaffold for the design of chemical processes. A sequential transition down the cascade ensures good results and enables the company to align the process requirements to the corresponding clinical development status.

- First, based on route finding or 'groundbreaking' chemistry, the chemical route to the target molecule is chosen.

- Second, in an initial optimization phase, a process draft is submitted for reagent screening; additionally, optimization with respect to maximum conversion is performed.

- Third, further optimization focuses on work-up and purification as well as the telescoping of steps.

- Fourth, the unit operations are refined and, during the scale-up, adaptations to the equipment are carried out.

- Fifth, for commercialization purposes, the process must be validated. A change control system guarantees that, prior to implementation, any process changes and improvements are carefully assessed with respect to product quality. 


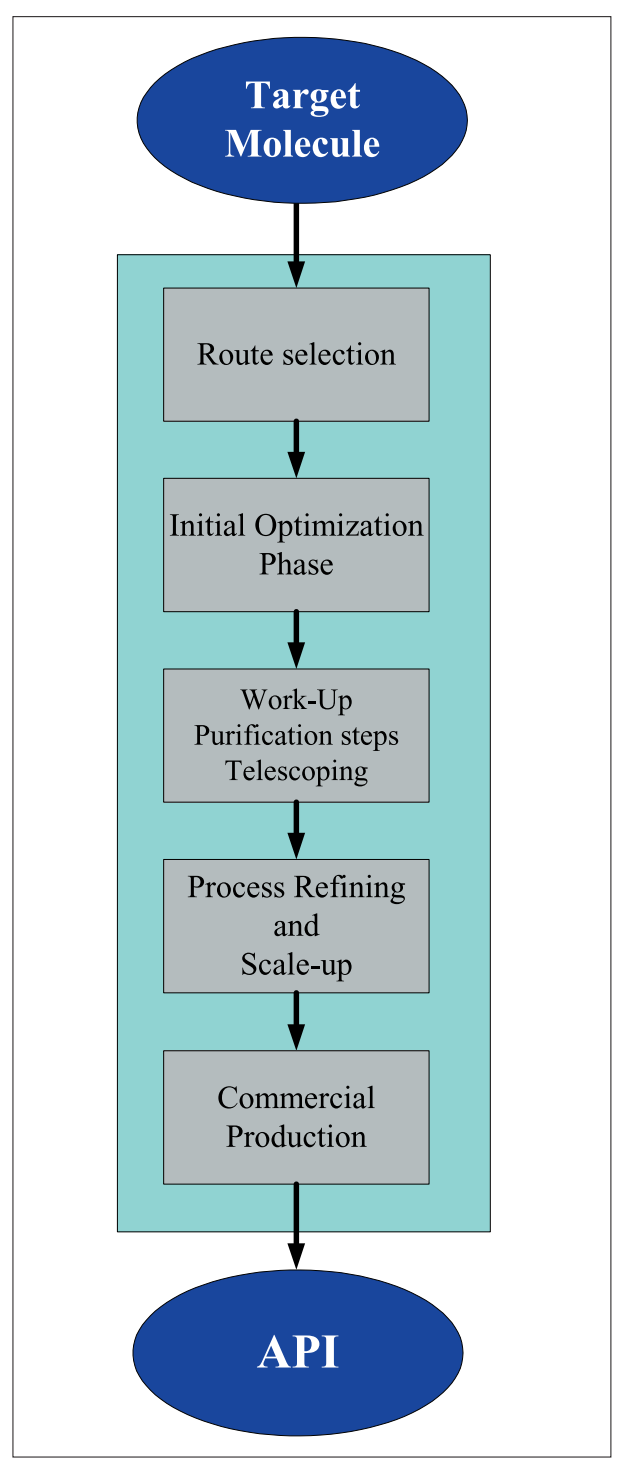

Fig. 2. Development cascade from the target molecule to the API

Special focus is placed on the physical properties of the API. When compared to the intermediates, the API's particle size distribution and crystal modification are parameters of particular attention. A separate section will address these aspects. Process development work is project teamwork. An efficient project management is thus a 'cornerstone' of process development. In the following sections, the dedicated specialists and project managers outline their contribution to the development of a new API synthesis, as carried out at Siegfried Ltd.

\section{Synthesis and Manufacturing Following a Developmental Cascade}

Route selection, choice of reagents for optimum conversion, establishing efficient work-up and purification steps, and telescoping are typically carried out on a lab-scale basis by the process chemist. Later process optimization (fine-tuning) is

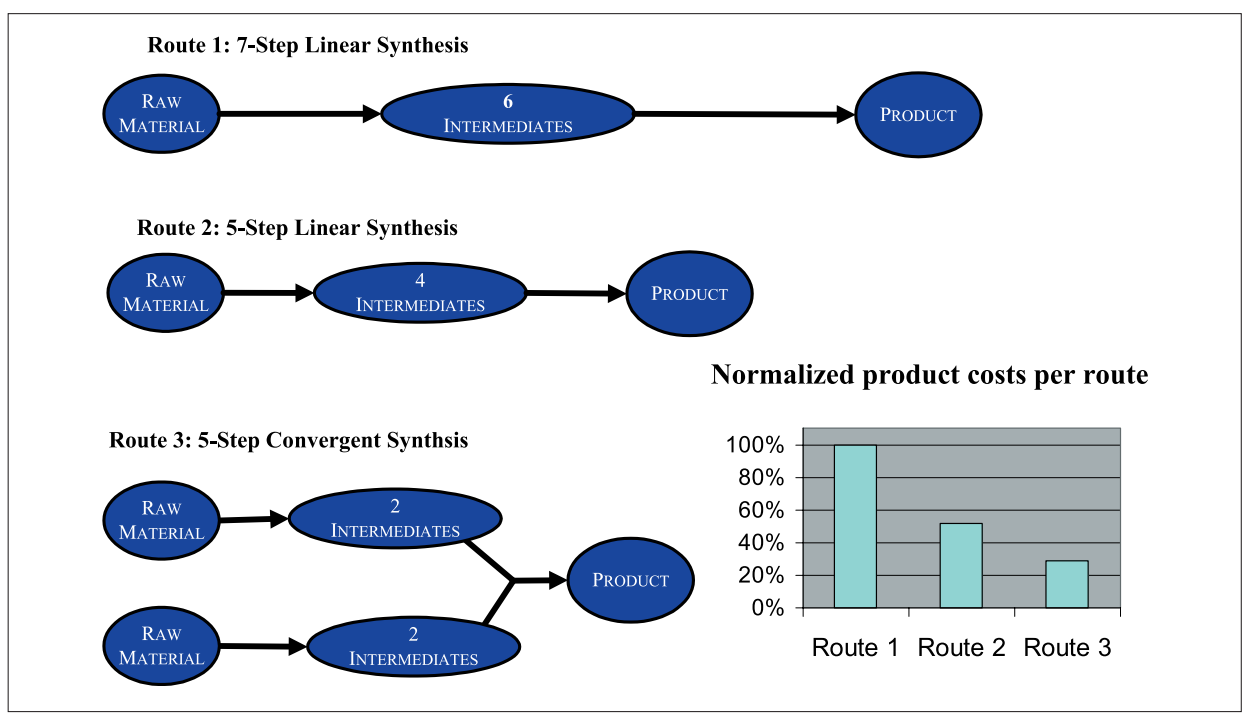

Fig. 3. Outline of the three hypothetical routes to a 'product'

performed at scale-up and, eventually, the final commercial process is executed and validated on a plant-scale.

\subsection{Route Selection}

Practical and expedient solutions worked out during the route selection phase determine the efficiency of a specific API synthesis. Routes used during research work are designed to enable the chemist to deliver a multitude of molecules requiring few adaptations to the methods. However, the route to the designated API molecule must deliver only the selected candidate. Based on information from global pharmaceutical companies, Gary Pisano (Harry E. Figgie, Jr. Professor of Business Administration, Harvard Business School) estimated that by changing from medicinal chemistry routes to adapted (second generation) routes, costs are reduced, on average, by $65 \%$ [2]. Second generation routes typically reduce the complexity [3][4] of the synthesis. This is achieved by

i) Reduction of the number of chemical transformations [4a];

ii) Splitting linear paths into convergent ones;

iii) By-passing inherently dangerous transformations [4b]; and

iv) The selection of low-cost raw materials.

By applying a simple cost model, one can demonstrate immediately how valuable shortcuts and optimizations can be [5]. As an example, the synthesis of a hypothetical product is presented. Using the MedChem route, a specific product is synthesized by a linear seven-step process. A second generation route forms the same product in five steps. The third process is a convergent route with five transformations as well. A cost analysis of these three hypothetical routes shows that shortening the linear synthesis from seven to five steps cuts the costs by approximately $50 \%$. If the linear synthesis can be split into two branches for a convergent synthesis, the costs can be reduced even more drastically by $75 \%$. (The assumptions for this model calculation are: $75 \%$ yield per step; the molecular weight is doubled from raw material to product; the intermediates have medium molecular weights; the raw material costs are $100 € / \mathrm{kg}$ and per transformation, $50 €$ per kg raw material/intermediate is assumed at any step; Fig. 3.)

As illustrated by this example, the specific route selection chosen exerts an enormous impact on the production costs of a molecule. The selected route is the foundation on which further optimization will be based and, therefore, receives top attention. Moreover, careful route selection affects other factors as well, such as flexibility in the manufacturing unit used and response time to increasing volume requirements.

\subsection{Initial Optimization Phase}

After selection of the synthesis route for the API molecule, the process must be optimized and fine-tuned. Each synthetic step has to be studied and optimized for best conversion conditions, and reagents are appropriately selected to improve the economy, quality, and ecological safety of the chosen route. Indeed, these later aspects (quality, safety, and ecology) should be designed into the process as early as possible. It is absolutely necessary to study and optimize each single synthesis step individually before they are cascaded together to define the ultimate process. Conversion factors, reagent type, availability and costs, solvent to reagent ratios, catalysts, reaction time, and solvents are studied at this stage of process development. By fine-tuning the reaction conditions, including temperature, stoichiometry, and reaction concentration (yield per volume), the synthesis can be 
optimized for best yield and purity. In addition to considering these conditions, the process chemist must also contemplate later stages of the developmental cascade while designing the process. This is important to minimize unit operations, permit telescoping, and ensure that waste streams are kept at a minimum. Because many of these parameters interact, specific tools, such as the statistical design of experiments (DoE) and lab automats, prove very helpful and effective. Using the DoE approach in process optimization results in a broad understanding of the influence of reaction parameters. The statistical analysis of such experiments helps to identify suitable and robust process parameters. The ultimate goal is to achieve the highest yield while providing the best quality possible. Modern DoE software, for example, STAVEX, visualizes the influence of the parameters for better process understanding and allows the process chemist to make reliable predictions within the multiparameter reaction room examined. With this, the chemist can then choose a robust region near the optimum. The output of this phase is a process selected to achieve the best conversion conditions. However, a chemical process is much more than simply a chemical conversion, and the subsequent steps may be more expensive than the initial chemical reaction.

\subsection{Optimization for Work-up, Purification Steps, and Telescoping}

After defining the conversion conditions for each synthetic step, the process is then checked for further cost improvements by optimization of the work-up conditions, re-use or regeneration of solvents, catalyst efficiency, raw material evaluation, and telescoping opportunities. Further, process safety and ecology are also addressed. The waste flows (gas, liquid, solid) are examined for waste treatment or disposal. For the work-up of a chemical reaction, it is often the case that more unit operations are necessary than for the conversion itself; this situation warrants a thorough investigation. A high purity achieved by complete conversion as output from the previous 'initial optimization' clearly helps to design a lean work-up. Knowledge of the work-up and purification procedure is as important as the reaction itself. Many so-called scaleup effects are caused by different work-up procedures between the lab and plant environments. For example, filtrations or phase separations that last five minutes in the lab may take several hours on a large scale. If the product is not stable in solution for this extended time period, the resulting product is not as pure as would be expected solely from the lab results. Filtrations are often switched from suction filtration to centrifugations, and washing of the cake changes from re-slurry to displacement washing.
Chromatography is a widely-used, highly-effective technique in the lab environment, but is expensive and impractical in the plant. For this reason, chromatography methods have to be replaced by techniques such as extraction, crystallization, precipitation or distillation. All these unit operations have to be carefully studied in order to obtain the knowledge required by the scale-up chemist. The behavior of plant equipment must be mimicked as closely as possible on a lab-scale. To simulate plant conditions, well-defined experiments (e.g. degradation kinetic studies under distillation conditions) can help avoid nasty surprises during the scale-up.

Because work-up and purification represent the majority of the unit operations, further optimization by telescoping of the intermediates has to be considered. If the purity of a reaction solution is sufficient for the next synthetic step, isolation of the product may be skipped. Telescoping is much more cost efficient than isolation of intermediates. Next, whenever wet material can be used for the subsequent step, time, money and dryer resources are saved. Nevertheless, whenever a process is telescoped, one must also consider the possible lost purification opportunity. In these cases, rework procedures should be anticipated and integrated into the process. In order to make prudent decisions concerning possible telescoping and the best point for 'quality up-grading', information on the purification efficiency of every unit operation and/or purification step is required. In general, a purification of an early intermediate is more cost efficient than one performed later in the synthesis; this is because every purification is associated with loss of yield and the product, of course, gets more expensive towards the end of a synthetic route. Keeping this in mind, it may be advantageous to add purification steps early in the synthesis, thereby allowing the telescoping of later steps. Often, the process finally implemented is a compromise among the yield, work-up, speed and quality. The key figure is the output of useful material per time and volume.

\subsection{Process Refining and Scale-up}

A scale-up is where the marriage between the chemical synthesis and the process technology takes place, and where the process chemist, the plant chemist, and the scale-up chemist meet and share their know-how. In the area of custom synthesis, the main objectives of the work in the scaleup area are:

- To produce the initial quantities of the final API material following cGMP (current Good Manufacturing Practice [1]) Guidelines for toxicological and clinical studies;

- To demonstrate the feasibility and the robustness of the chemical synthesis on a larger scale;
- To evaluate and define the equipment and the process technology that are needed for a large scale production;

- To identify additional (equipment-related) critical parameters [6].

In past years at Siegfried, the only way to gather all this information was a scaleup procedure involving three steps: First, the synthesis was run in the kilo-lab-scale (typically in 60-litre reactors). The data generated resulted in a re-assessment of the process. In a second step, the findings and process improvements of the kilo-lab campaign were scaled further to the pilot-scale (typically, in 250-litre reactors). Third, after a thorough check of the synthesis parameters and the chosen technical solutions, the process was implemented and validated in the production-scale (ranging from 1600 to 4000-litre reactors). Bowing to pressure to shorten the time to market, the following questions are frequently posed today: What do we learn by following this three-step model: kilo-lab, pilot plant, production? Is it possible to skip the kilo-lab or the pilotplant step? These questions cannot be answered on a general basis. But it is quite evident that all the relevant questions for the scale-up of a process must be addressed by the process chemist in an early stage of process development ( $c f$. section 2.3.). Process knowledge should be generated on a smaller scale. Indeed, the better a process is understood, the lower the probability of encountering unexpected 'scale-up effects'. A close and fruitful cooperation between the process chemist and the scale-up chemist is thus a key factor for the fast and successful introduction of a new, robust and economical large-scale chemical process.

\subsection{Commercial Production Aspects}

Entering the commercial production phase, the process is 'fixed' by filing the methods used with the health authorities. Standard operating ranges, as defined during the earlier phases, are applied and the process is 'frozen'. Some adaptations to the plant equipment are done in the initial batches. Parameters for filtration, centrifugation, distillation, phase separation, and drying are adjusted to the equipment used. To demonstrate reproducibility, the process is validated once these adaptations are done. Validation, as executed by Siegfried, means that at least three consecutive batches are run under the standard operating conditions. These must meet the predefined acceptance criteria for the critical process parameters, quality, and yield. Hence, the quality parameter (Fig. 1) is addressed.

Inherent safety and robustness have to be built into the process, before as well as during the process introduction at the plant. The plant manager is responsible for addressing the safety aspects, such as work 
hygiene and house-keeping. As mentioned above, the output per time and volume determines the costs of a production. The best use of the reactor configuration allows for parallel runs of several batches in one reaction train (assembly of several reactors), leading to shorter lead times and lower costs. Nevertheless, even once the process is running on plant-scale, cost-saving improvements may be considered, even those that exceed the boundaries of the validated process. Such ideas are subject to a costbenefit analysis and are handled under the strict guidance of a change control system: impact on quality, registration dossier and plant operations are considered.

\subsection{Process Development for Physical Properties of Solid Forms}

With respect to stability and bioavailability, it is the physical properties of the drug substance that determine the behavior of the drug product (formulated product). It is quite often the case that API candidates are acids or bases from which salts can be formed. This allows one to select the salt candidates that exhibit the desired properties (Fig. 4). This process can be started as

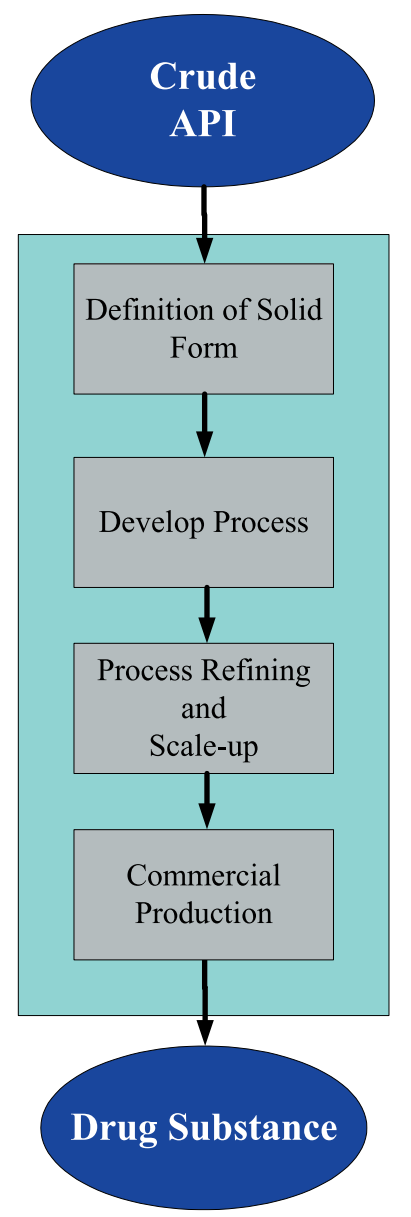

Fig. 4. Cascade for the development of the solid form. Focus is on crystal modification, purity, and yield

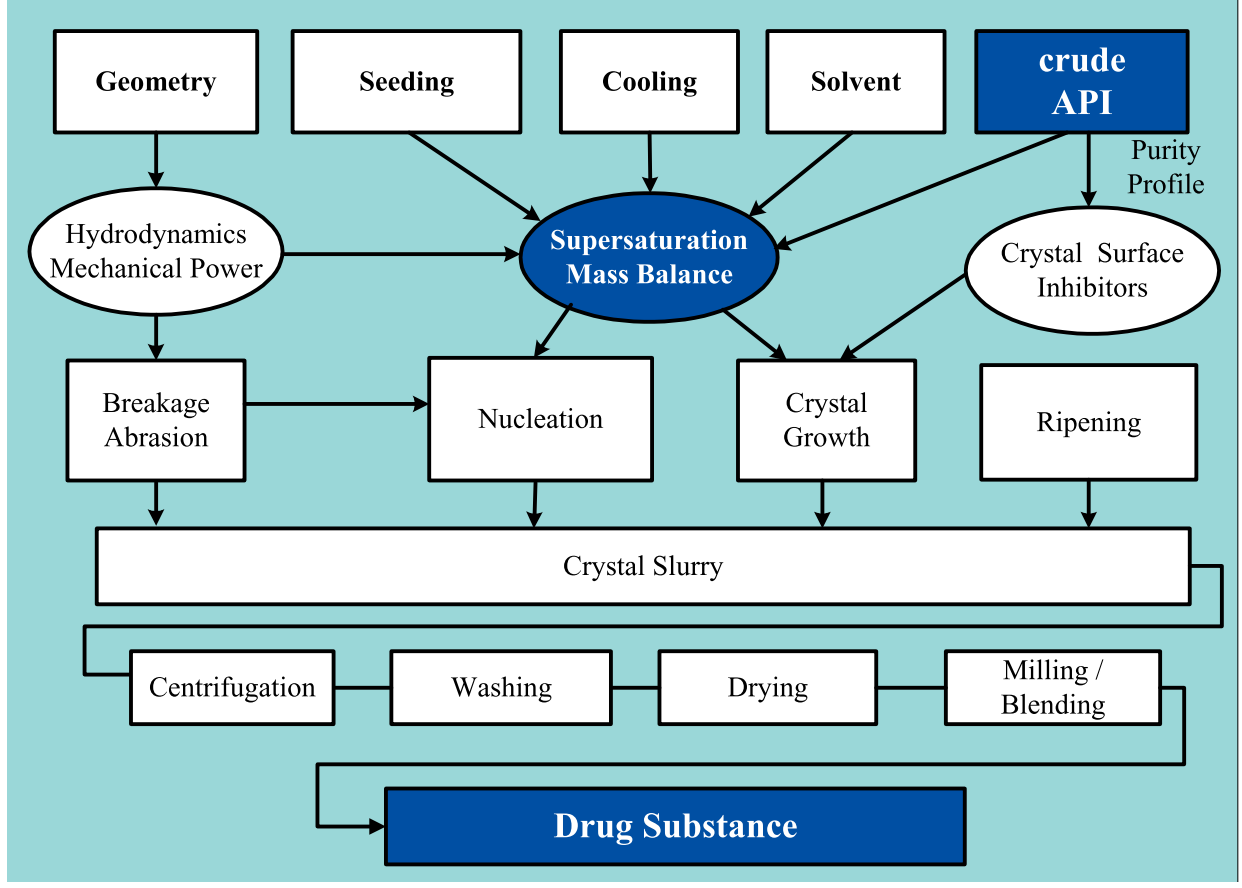

Fig. 5. From the crude API molecule to the drug substance by crystallization

soon as the initial quantities of the API become available.

Selection of a salt for the drug substance is done in collaboration with the pharmacology experts and based on dissolution, stability, flowability, bulk density, compressibility, kinetic and thermodynamic data.

One important technique for the production of solids showing the desired physical properties, such as crystalline form and particle size distribution (PSD), is the crystallization process which is then followed by the unit operations: solid liquid separation (centrifugation), washing, drying, milling, and blending [7]. Batch crystallization is most common in API manufacturing. The crystallization process utilizes the principle of supersaturation (Fig. 5). The factors that influence this supersaturation are determined on lab-scale. Information about solubility curves, meta-stable regions and kinetic data are addressed, as are cooling rates and solvents. Furthermore, the geometry of the agitator and reactor, the seeding temperature, the amount and the PSD of seed material, and even the way the seeds are added (slurry or powder), determine the behavior of the supersaturation phase. The impact of impurities should not be underestimated; these might act as inhibitors for crystallization and can lead to different crystal habits. Once the crystallization process has started, abrasion of initial crystals or spontaneous nucleation can form new seeds and secondary nucleation starts. Crystal growth and ripening are the dominant steps in the later stage of the crystallization process.

Centrifugation, washing, drying, milling, and blending finish the process and lead to the drug substance with all its physical properties.

All these technical operations have to be examined, understood and scaled, as is undertaken with the chemical process. Largescale adjustments, due to, say, the geometry of the equipment used, are common. In one specific challenging case, we even had to use the principle of statistical design of experiments on a milling train to find the optimal parameters to meet a very tight PSD specification [7].

\section{Supportive Experts}

\subsection{Analytical Aspects}

Analytical chemistry forms an important success factor in the development of an ideal manufacturing process. The effort expended for the analytical development of an API is about one-third of that invested in the chemistry. Analytics are the development chemist's eyes with which he 'sees' the quality aspects of raw materials, intermediates and products, the composition of reaction mixtures and the whereabouts of individual by-products, so-called impurity tracking.

During the route selection phase, the main analytical focus is on generating qualitative and semi-quantitative information about the targeted new molecules. Fast and sound analytical information may tip the balance toward a favorable route. Sophisticated analytical techniques frequently used in this phase include NMR, MS, GC/ MS or HPLC/MS. As 'only' a rough value of the purity and the substance quality is required, even simpler methods, such as TLC 
(thin layer chromatography) or standardized HPLC, can be useful.

As the project progresses down the developmental cascade, the requirements demanded of analytics change and increase. Product quality must be determined and specified: assay of the main product, determination and quantification of related substances for every chemical step, impurities tracking. This task requires well-developed methods of chromatography. Since impurities are often only found in minor quantities, their structure is routinely determined by coupled methods, e.g. LC-MS.

For the analysis of the API, in addition to assay and purity-determining and identification methods, further techniques provide information about the physical properties of the API. Particle size distribution is determined by specific techniques, such as microscopy, sieve analysis, or laser diffraction; crystal modification is analyzed by XRPD (X-ray powder diffraction), DSC (differential scanning calorimeter), IR (infra red) or RAMAN analysis. The interpretation of the analytical data provides the basis for the justification of specifications (raw materials and intermediates), or the decision of whether an intermediate has to be isolated or purified. These are important factors that exert a direct impact on costs and robustness of a manufacturing process. The investment in analytics during the development pays off when a product comes onto the market.

Just as with the chemical processes, analytical methods have to undergo optimization. Once the process is fixed (in Stage 3 of the developmental cascade), the analytical methods are then optimized for their intended use. In this third stage of the project, a substantial analytical effort is necessary. The cycle time of in-process controls may be limiting and GC or HPLC run-times are therefore reduced to a justifiable minimum. Fast analytical methods, such as the UPLC or fast GC technique, help shorten cycle times and increase plant efficiency. Analytical method optimizations must be performed without a loss in the quality of the results. Finally, this is proven by using customized and validated methods for the release of critical raw materials, key intermediates and the API.

\subsection{Raw Materials and Reagents \\ - Sourcing Aspects}

During the development of an API, the provision of raw materials and reliable suppliers is essential for costing, quality and speed. Route selection may be influenced by the availability of a certain starting material.

The three criteria, time, costing, and quality, often conflict. To achieve a balance among these three, sourcing activities are assigned different depth levels, depending on the stage of the project. Siegfried uses three modules for sourcing:

i) Screening for price, based on existing data and information;

ii) Price and availability, including the evaluation of suppliers, offers, delivery times and specifications;

iii) Full evaluation, including packaging, capacities, material safety data sheets (MSDS), etc.

The full evaluation leads to a reliable and cost-effective partnership with suppliers who deliver at optimum conditions.

A sound partnership between the buyer and supplier is the key to success and to improving the level of efficiency throughout the entire supply chain. More and more, purchasing is being made from low cost countries. In order to execute global sourcing decisions, one must also consider that there is more risk and more work required to manage logistic and quality aspects.

The total cost of ownership (TCO) arises not only from the purchase price. Additional sourcing costs are generated by: prospecting new suppliers, selecting a supplier, sampling and analytical testing, qualifying procedures, PO placement, taxes and duties, transportation, inventory considerations, regulatory considerations, and further costs for non-compliant material. Hence, we have to be aware of not only the visible part of the TCO iceberg (Fig. 6) that is on the radar screen, but also all the 'hidden costs' below the (water) surface.

\subsection{The Role of Quality Assurance}

Quality is basically the accordance of a result with pre-defined requirements and expectations due to an intended use. Thus, throughout the development and commercial application of an API for use in humans, cGMP-related (sometimes countryspecific) quality regulations [1] must be applied. However, at the start of API development - compared to commercial produc-

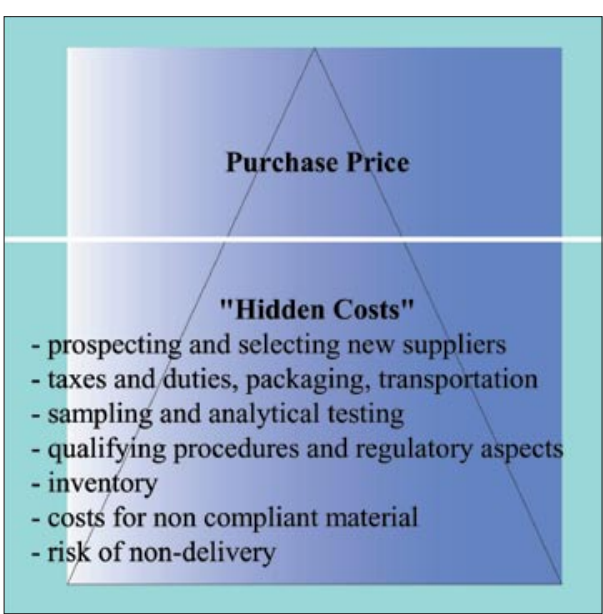

Fig. 6. Total Costs of Ownership (TCO)

tion - limited information is available, e.g. only the general route of synthesis.

Knowledge of where to purify, purities sufficient for further processing and the like, have to be generated. Reagents/solvents can still vary, physical and chemical parameters and their influence on quality need to be determined, etc. This results in a bi-directionally increasing GMP level: in one direction, by moving from the clinical trial phases I-III to commercial production, and, in a second direction, by moving from starting materials via API starting material towards the final API (Fig. 7).

To fulfill the quality requirements for APIs for human use, as defined in the guidelines, development has to generate specific data, such as:

- Quality attributes of raw materials (necessary for successful API synthesis);

- Critical process parameters (due to the large influence on API quality);

- Impurity tracking throughout all steps of synthesis (basis for definition of API starting material(s), adequate specifications and purification steps);

- Purity profiles of intermediates and API;

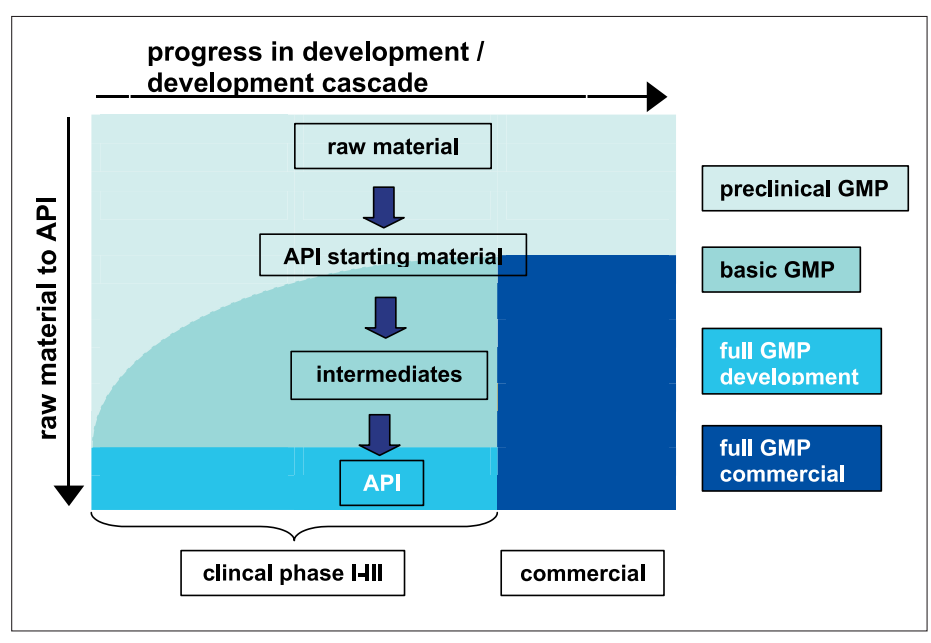

Fig. 7. Increasing GMP levels in development and synthesis 
- Adequate in-process and analytical limits for process and batch quality control, etc.

From the quality assurance point of view, the final outcome of development work must be an optimized, controllable process ready for process validation. It has to guarantee a reproducibly successful production of the API. The accrued information is summarized in a comprehensive development report, including sound rationales and/or experimental proof for quality-relevant decisions. Finally, it is very important to have, at all times, from the start of API development to commercial production, an appropriate quality concept and strategy in place. It is mandatory to have extensive process knowledge available should one wish to define the API starting material at a later step in the synthesis [8]. This limits the application of full GMP to the last chemical transformations. Cost reductions due to a higher flexibility in the production of the pre-API starting material steps are possible. Considered from this angle, QA's requirements do not place additional hurdles on the chemists, but keep flexibility in the production sequence. However, within the GMPcontext, all decisions should be carefully evaluated with respect to quality aspects. Quality Assurance should provide intensive guidance, because failing in quality means failing on the market.

\subsection{Registration Aspects}

The chemical process is then eventually filed. The sum of the activities described so far generates the basis for a registration dossier.

From the regulatory point of view, a huge evolution has taken place since the 1970s, i.e. a change from simple API specifications towards a science-driven approach for the manufacturing of a defined API quality.

This also impacts the developmental process of the API from the beginning. Knowledge gleaned from development identifies the process and provides the scientific understanding of the accompanying critical specifications and manufacturing controls, which in the end, result in a quality API. Critical parameters and ranges necessary for the reproducible production of an API, which meet the predefined quality attributes, are identified during the development phase. Specific development efforts include identifying process parameters that could affect the critical quality attributes of the drug substance, and determine the range for each critical process parameter expected to be used during routine manufacturing and process control. These critical parameters are included in the regulatory filing and the knowledge serves as background information for regulatory inspections and future process optimization, $c f$. section 2.5. The knowledge gained during development

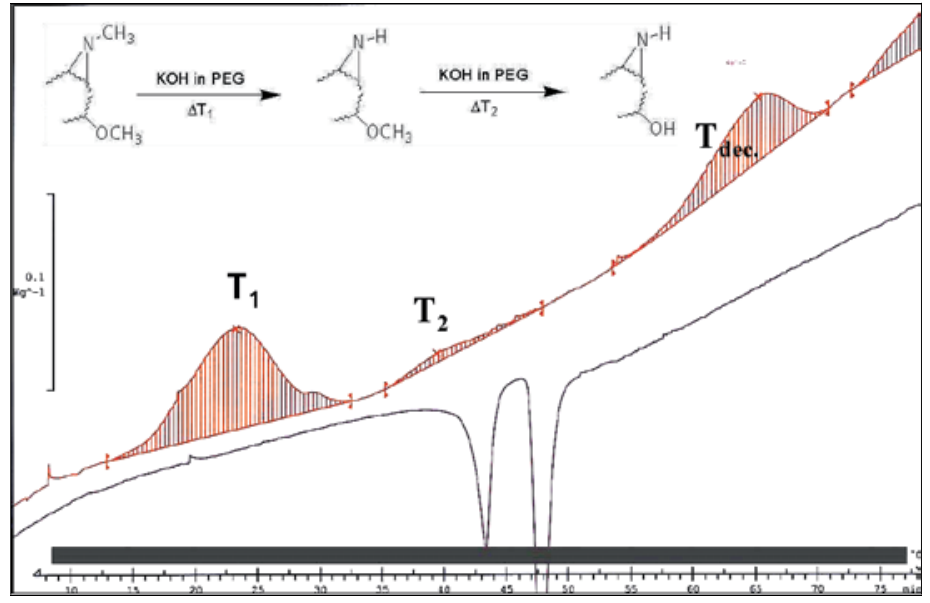

Fig. 8. Example of a DSC. Based on this information the optimum conditions for the sequential and selective de-methylation were set.

provides the basis for science-based submissions, as expected by the harmonized pharmaceutical quality system, i.e. $\mathrm{ICH}$ guidelines Q9, Q10 and soon Q8C [8][9].

\subsection{Safety, Health and \\ Environmental Aspects}

In chemical processes, one strives to avoid uncontrolled heat and pressure releases. Operators should be trained in the proper handling of harmful substances, and their exposure to such must be minimized. Siegfried's Safety, Health and Environmental (SHE) department has established a systematic program that guarantees early detection of hazards originating from these sources.

Heat flow management, thermal stability, and gas evolution are measured on lab-scale using DSC, DTA, and RC1 techniques. These analyses are performed during the development of the process, thus allowing modifications should a safety issue be identified. Heat-flow regime information and possible run-away scenarios show where reagent accumulation could occur, where decomposition reactions may occur, and what kind of plant equipment might be suited for scale-up. With this information in hand, the processes can be optimized with respect to safety and stability. An example for this is the sequential de-methylation of an N,O-dimethyl compound. Based on the DSC figures measured during the process design phase (Fig. 8), optimum conditions for the two de-methylation steps could be identified, thereby avoiding undesired degradation. The critical nature of substances must be evaluated right from the beginning of the development activities. For commercially available reagents, toxicity and safety data are on-hand. For new intermediates, an initial assessment on how toxic a compound may be is done by a hazard estimation based on structural elements [10]. Using these results, adequate protective measures for a safe handling can then be defined. In addition, the process chemist may modify the synthesis in such a way that dangerous intermediates are not isolated at all.

Waste treatment is the third aspect of SHE. For organic streams, the most ecofriendly version is the direct recycling into the process. If this is not possible, alternate uses are evaluated, such as its use as fuel in the cement industry or in the boiler house. Here, again, there is feedback to the process chemist: Solvents, such as low alcohols, acetone, even toluene, are easy to handle, while others, e.g. chlorinated compounds, require disposal/incineration at a specialized company and thus increase costs. For streams of inorganic solids, the situation is similar: re-use is better than disposal, and if disposal is necessary, a minimum load of critical components (e.g. heavy metals) should be present.

The same principle applies to gases. At Siegfried, a 'waste' gas can sometimes be directly used as a processing reagent in the same process.

Wastewater streams evoke the largest volumes that have to be handled; the goal is to avoid their formation, but in many cases, this is not possible. Wastewater streams are best treated with biological degradation in the regional wastewater treatment plant. If biodegradability is not an option, physical and/or chemical treatment is required. Again, feedback to the process chemist is most important in finding the best solution considering both ecological and economical factors.

\subsection{Project Management}

Project management is the common term applied to all instruments used for planning, controlling, and execution of projects. In the development of chemical processes, these instruments are applied to coordinate the activities of the many departments involved. The methods focus on promoting inventive solutions and forming 


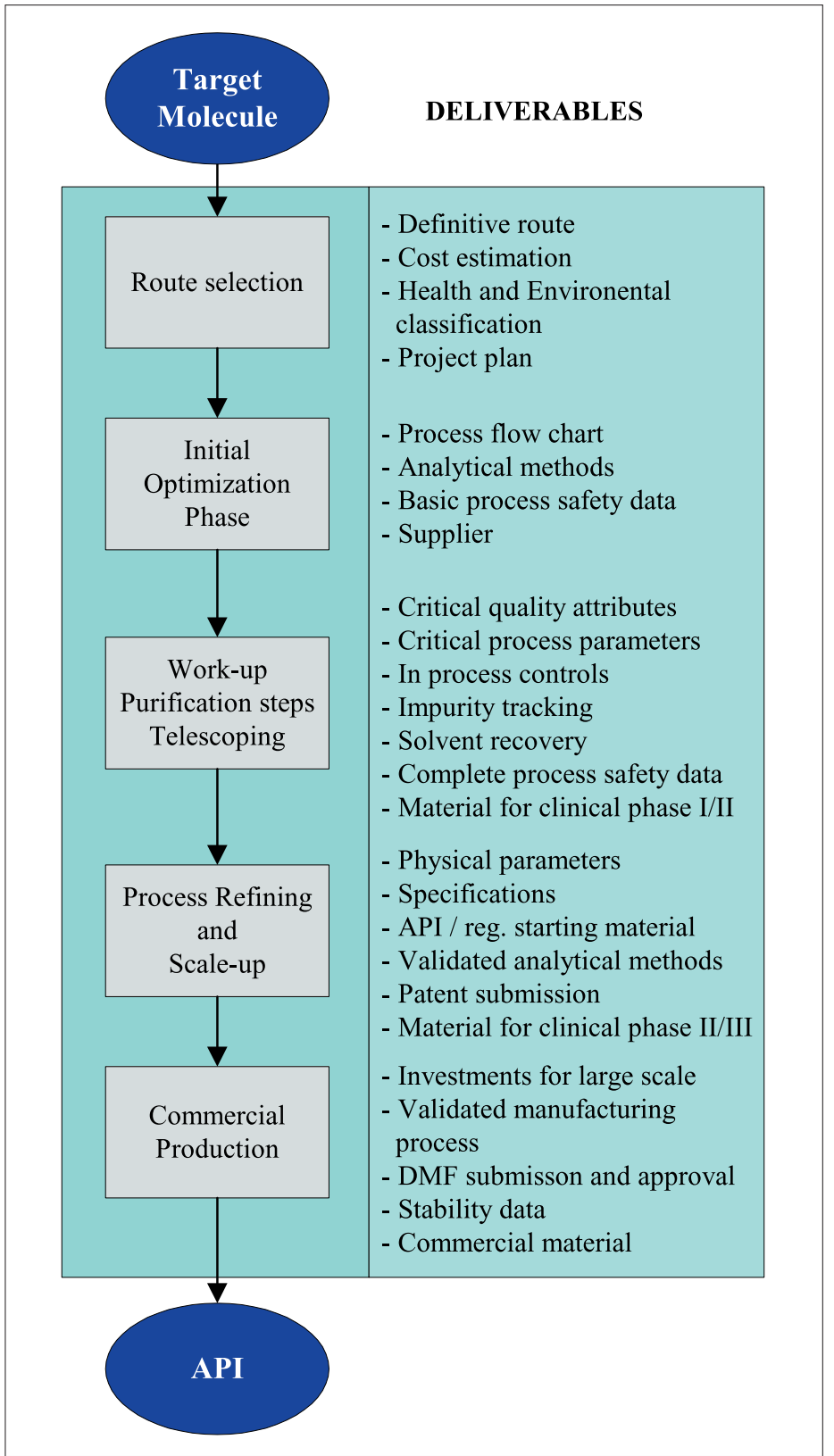

Fig. 9. Deliverables for the developmental cascade
[1] ICH Guidelines, Food and Drug Administration (FDA) Code of Federal Regulation 21 CFR 201, 211; EU-GMP Regulations, Pharmacopoeias of Europe (EP), USA (USP). Britain (BP), Japan (JP).

[2] G.P. Pisano, 'The Development Factory', Harvard Business School Press, Boston, 1996.

[3] N.G. Anderson, 'Practical Process Research \& Development', Academic Press, London, 2000.

[4] An excellent source for examples is $O r$ ganic Process Research and Development. Illustrative examples are a) P.J Harrington, H.N. Khatari, B.S. DeHoff, M.R. Guinn, M.A. Boehler, K.A. Glaser, Org. Process Res. Dev. 2002, 6, 120; b) S.G. Ruggeri, D.R. Bill, D.E. Bourassa, M.J. Castaldi, T.L. Houck, D.H. Brown Ripin, L. Wei, N. Weston, Org. Process Res. Dev. 2003, 7, 1043.

[5] H.R. Marti, S. Peterli, sp2 2003, 2, 22

[6] H.R. Marti, $s p 2$ 2004, 3, 35.

[7] M.K. Levis, Chemanager Europe 2006, 1, 8; b) S. Feiler, M.K. Levis, P. Solot, cav Chemie Anlagen + Verfahren 2006, 4, 74 .

[8] W.P. Ganzer, J.A. Materna, M.B. Mitchell, L.K. Wall, Pharm. Technol., 2005, 29, website: http://www.pharmtech.com/ pharmtech/content/printContentPopup. jsp?id=170114.

[9] 'The Gold Sheet', F-D-C Reports, Ed. B. Paulson, 2005, 39, 1.

[10] Databases used by Siegfried are: TOXNET, RTECS, EMEA, AGCIH; proven to be versatile tools. teams that work together efficiently. Typically, the project management activities increase with the progress and the complexity of a project. Without the methods and tools provided in project management, the development of chemical processes would be unfocused, unstructured, inefficient, and result in overshooting costs and timelines. To implement project management, traditionally organized companies change to a matrix organization where project managers lead the technical experts in teams next to the line management. The structure of chemical process development projects is similar to other types of projects, although the deliverables for each project phase are, of course, specific for API developing manufacturing processes (Fig. 9).

Developing innovative processes within a short time-frame also involves taking some risks. Project management provides instruments to manage these risks and to support the teams in avoiding mistakes.

\section{Conclusion and Experiences}

Process development is much more than simply optimizing the process to the needs of 'ideal chemistry'. Various specialists are involved. To keep all activities focused and well-organized, a developmental cascade provides a valuable scaffold. Even in cases where not all elements are used to their full extent, it nevertheless helps to structure the work. Teamwork is essential and a strong project manager enables the organization to make optimal use of the usually restricted resources.

Received: June 18, 2006 